\title{
COVID-19 pandemic impact on uro-oncological disease outcomes at an Italian tertiary referral center
}

\author{
Marco Oderda ${ }^{1}$ (1) - Francesco Soria ${ }^{1} \cdot$ Francesco Rosi $^{1} \cdot$ Giorgio Calleris $^{1} \cdot$ Simone Mazzoli ${ }^{1}$. Andrea Giordano ${ }^{1}$. \\ Giuseppe Pizzuto ${ }^{1}$. Alessandro Marquis ${ }^{1} \cdot$ Matteo De Bellis $^{1} \cdot$ Federico Vitiello $^{1}$ - Eugenia Vercelli ${ }^{1}$. \\ Federica Peretti ${ }^{1}$. Gabriele Montefusco ${ }^{1}$. Paolo Gontero ${ }^{1}$
}

Received: 20 July 2021 / Accepted: 15 September 2021 / Published online: 25 September 2021

(c) The Author(s), under exclusive licence to Springer-Verlag GmbH Germany, part of Springer Nature 2021

\begin{abstract}
Purpose To assess differences in referral and pathologic outcomes for uro-oncology cases prior to and during the COVID pandemic, comparing clinical and pathological data of cancer surgeries performed at an academic referral center between 2019 and 2020.

Methods We collected data of 880 prostate biopsies, 393 robot-assisted radical prostatectomies (RARP) for prostate cancer (PCa), 767 trans-urethral resections of bladder tumor (TURB) and 134 radical cystectomies (RC) for bladder cancer (BCa), 29 radical nephro-ureterectomies (RNU) for upper tract urothelial carcinoma, 130 partial nephrectomies (PN) and 12 radical nephrectomies (RN) for renal cancer, and 41 orchifunicolectomies for testicular cancer. Data of patients treated in 2019 (before COVID-19 pandemic) were compared to patients treated in 2020 (during pandemic).

Results No significant decline in uro-oncological surgical activity was seen between 2019 and 2020. No significant increase in time between diagnosis and surgery was observed for all considered cancers. No differences in terms of main pathologic features were observed in patients undergoing RARP, TURB, RNU, RN/PN, or orchifunicolectomy. A higher proportion of ISUP grade 3 and 4 PCa were diagnosed in 2020 at biopsy $(p=0.001)$, but this did not translate into worse pathological grade/ stage at RARP. In 2020, more advanced disease features were seen after RC, including lymph node involvement $(p=0.01)$ and non-organ confined disease $(p=0.02)$.

Conclusion Neither decline in uro-oncologic activity nor delay between diagnosis and treatment was observed at our institution during the first year of COVID-19 pandemic. No significant worsening of cancer disease features was found in 2020 except for muscle-invasive BCa.
\end{abstract}

Keywords Uro-oncology $\cdot$ Covid-19 $\cdot$ Upstaging $\cdot$ Cancer $\cdot$ Pathological outcomes $\cdot$ Delay

\section{Introduction}

The rapid spread of coronavirus disease 2019 (COVID-19) throughout the world has had dramatic effects on healthcare systems. The hospitals have become quickly overwhelmed and a reallocation of medical resources has been made necessary to face the crisis [1]. As a consequence, the level of activity of medical disciplines not primarily involved in the management of COVID-19 patients has reduced and

Marco Oderda

marco.oderda@libero.it

1 Division of Urology, Department of Surgical Sciences - Urology, Città della Salute e della Scienza di Torino Molinette Hospital, University of Turin, Turin, Italy all "non-urgent" procedures have been postponed. Urooncological consultations and surgeries have also reported a dramatic reduction, raising concerns about the risks of adverse oncologic outcomes related to delayed diagnosis and/or treatment $[2,3]$. Several recommendations have been recently published to guide the management of urological conditions during these troubled times [4-6]. In the meantime, evidence has accumulated supporting the idea that most uro-oncologic elective surgeries can be safely postponed when the availability of health care resources is limited [7]. However, concerns remained on the possibility that COVID-19 pandemic-related constraints on healthcare access might translate into more advanced disease features [6]. Given the prolongation of the current crisis, the centralization of uro-oncological surgeries in "COVID-19-free" 
tertiary urological centers could be a smart alternative to guarantee a timely, high-quality, and effective treatment of genitourinary cancer patients $[3,8]$.

Aim of this study was to evaluate if COVID-19 pandemic has actually led to more advanced disease features in urological cancers, including prostate cancer (PCa), bladder cancer (BCa), upper tract urothelial carcinoma (UTUC), renal cancer, and testicular cancer at an academic referral center.

\section{Patients and methods}

Institutional review board approval was obtained before the initiation of the study. Patients treated for suspected or confirmed urologic cancer between January 2019 and December 2020 at a single academic referral center (Division of Urology, Department of Surgical Sciences, University of Torino) were included. In detail, we collected baseline data and tumor-related features of patients who underwent prostate biopsy and/or robot-assisted radical prostatectomy (RARP) for PCa, trans-urethral resection of bladder tumor (TURB) and/or radical cystectomy ( $\mathrm{RC}$ ) for $\mathrm{BCa}$, radical nephroureterectomy (RNU) for high-risk UTUC, partial nephrectomy (PN) or radical nephrectomy (RN) for renal cancer, and orchifunicolectomy for testicular cancer. Moreover, the time between the occurrence of symptoms/diagnosis and surgery was evaluated. Only patients with complete data regarding the variables of interest were retained for the analysis.

\section{Statistical analysis}

The primary endpoint of the study was to assess differences in referral and pathologic outcomes for uro-oncology cases prior to and during the COVID pandemic. We hypothesized that COVID-19 pandemic might have led to a shift towards higher pathological tumor stage/grade, possibly due to a delay in diagnosis and/or surgical schedule. To test our hypothesis, data of patients treated in 2019 (before COVID19 pandemic) were compared to those of patients treated in 2020 (during COVID-19 pandemic). A comparative analysis by trimester was performed for all evaluated procedures except for RC, since all patients receiving $\mathrm{RC}$ underwent surgery $\leq 30$ days from the time of surgical indication.

Categorical variables were reported as absolute numbers and proportions while continuous variables as medians with interquartile ranges (IQR) or means with standard deviation (SD) when appropriate. Chi-square and Mann-Whitney $U$-tests were performed for categorical and continuous variables to compare the populations, respectively. Statistical analyses were performed using STATA 13 (Stata Corp., College Station, TX, USA). All tests were two-sided and $p<0.05$ was considered as statistically significant.

\section{Results}

\section{Prostate cancer}

Overall, 880 patients received prostate biopsy for suspicion of PCa. Among these, 414 (47\%) underwent biopsy in 2019 and 466 (53\%) in 2020, respectively (Suppl. Table 1). A higher number of patients received pre-biopsy magnetic resonance imaging (MRI) ( $84 \%$ vs $75 \%, p=0.002)$ and subsequent fusion biopsy ( $73 \%$ vs $64 \%, p=0.003$ ) in 2020 compared to 2019. Notably, a higher proportion of ISUP grade 3 and 4 and a lower proportion of ISUP grade 2 was observed in 2020 compared to $2019(p=0.001)$.

After confirmation of PCa, RP was performed in 207 (54\%) and 186 (47\%) patients in 2019 and 2020, respectively (Table 1, Fig. 1A). In 2020, a higher proportion of robotic-assisted (97\% vs $90 \%, p=0.008$ ) and nerve-sparing procedures ( $71 \%$ vs $62 \%, p=0.04$ ) was observed. With regard to pathological findings, a lower rate of seminal vesicle invasion ( $8 \%$ vs $15 \%, p=0.02$ ) and positive surgical margins ( $8 \%$ vs $20 \%, p<0.001$ ) was reported in 2020 as compared to 2019. However, no difference in terms of tumor stage, ISUP grade, and lymph node involvement was found.

When focusing on the time between PCa diagnosis and definitive surgery, no difference was reported between 2019 (median of 102 days) and 2020 (median of 105 days) (Fig. 1B). Eleven patients were postponed due to COVID-19 positivity ascertained during preoperative work-up. After diagnosis of low-risk PCa, 27 patients were addressed to active surveillance in 2019 and 18 in 2020.

\section{Bladder cancer}

Overall, 767 patients underwent TURB for suspected BCa. Among these, 407 (53\%) and 360 (47\%) were treated in 2019 and 2020, respectively (Fig. 1C). Baseline patients' and tumor's characteristics are depicted in Table 2. No difference in tumor stage/grade between patients treated in 2019 and 2020 was reported. Similarly, the time between the occurrence of symptoms and TURB did not significantly differ over the study period (Fig. 1D).

$\mathrm{RC}$ was performed in $58(43 \%)$ and $76(57 \%)$ patients in 2019 and 2020, respectively (Fig. 1G). Notably, a shift towards a higher rate of lymph node involvement $(16 \%$ in 2019 vs $36 \%$ in $2020, p=0.01$ ) and non-organ confined disease ( $55 \%$ vs $74 \%, p=0.025$ ) at surgery was observed during the study period (Table 3 ). The time between the indication for surgical treatment (after TURB or at the completion of neoadjuvant chemotherapy) and RC did not differ between groups and remained within 30 days in all cases. 
Table 1 Descriptive characteristics for the cohort of 393 patients treated with radical prostatectomy between January 2019 and December 2020

\begin{tabular}{|c|c|c|c|}
\hline \multirow[t]{2}{*}{ Variables } & \multicolumn{2}{|c|}{$\begin{array}{l}\text { Year of radical prostatec- } \\
\text { tomy }\end{array}$} & \multirow[t]{2}{*}{$p$ value } \\
\hline & 2019 & 2020 & \\
\hline Number of patients, $n(\%)$ & $207(53)$ & $186(47)$ & \\
\hline Age, median, years (IQR) & $68(62-72)$ & $69(62-72)$ & 0.2 \\
\hline PSA, mean (SD) & $10.2(12.7)$ & $10.0(14.4)$ & 0.9 \\
\hline MRI execution, $n(\%)$ & 191(92) & $169(92)$ & 0.5 \\
\hline \multicolumn{4}{|c|}{ Clinical tumor stage (with MRI or DRE), $n(\%)$} \\
\hline $\mathrm{cT} 1$ & $18(9)$ & $13(7)$ & 0.8 \\
\hline cT2 & $137(66)$ & $125(67)$ & \\
\hline сT3a & $26(13)$ & $27(15)$ & \\
\hline $\mathrm{cT} 3 \mathrm{~b}$ & $6(3)$ & $3(2)$ & \\
\hline cT4 & $0(0)$ & $1(1)$ & \\
\hline Robotic approach, $n(\%)$ & $187(90)$ & $180(97)$ & 0.008 \\
\hline Nerve-sparing surgery, $n(\%)$ & $129(62)$ & $130(71)$ & 0.04 \\
\hline \multicolumn{4}{|l|}{ ISUP grade at RP, $n(\%)$} \\
\hline 1 & $1(0)$ & $1(0)$ & 0.1 \\
\hline 2 & $94(46)$ & $72(39)$ & \\
\hline 3 & $72(35)$ & $81(44)$ & \\
\hline 4 & $15(7)$ & $20(11)$ & \\
\hline 5 & $24(12)$ & $12(6)$ & \\
\hline \multicolumn{4}{|l|}{ Pathological tumor stage, $n(\%)$} \\
\hline pT2a & $20(10)$ & $14(7)$ & 0.2 \\
\hline pT2b & $9(4)$ & $14(7)$ & \\
\hline pT2c & $83(40)$ & $87(47)$ & \\
\hline pT3a & $63(30)$ & $56(30)$ & \\
\hline pT3b & $31(15)$ & $15(8)$ & \\
\hline pT4 & $1(1)$ & $0(0)$ & \\
\hline $\begin{array}{l}\text { Extracapsular extension, } n \\
(\%)\end{array}$ & $93(45)$ & $71(39)$ & 0.1 \\
\hline $\begin{array}{l}\text { Seminal vesicle invasion, } \\
n(\%)\end{array}$ & $31(15)$ & $15(8)$ & 0.02 \\
\hline \multicolumn{4}{|l|}{ Nodal tumor stage, $n(\%)$} \\
\hline No & $106(51)$ & $110(59)$ & 0.3 \\
\hline $\mathrm{N}+$ & $24(12)$ & $17(9)$ & \\
\hline $\mathrm{NX}$ & $77(37)$ & $59(32)$ & \\
\hline $\begin{array}{l}\text { Positive surgical margins, } \\
n(\%)\end{array}$ & $42(20)$ & $15(8)$ & $<0.001$ \\
\hline $\begin{array}{l}\text { Time from diagnosis to treat- } \\
\text { ment, days, median (IQR) }\end{array}$ & 102(79-139) & 105(72-141) & 0.9 \\
\hline
\end{tabular}

\section{Upper tract urothelial carcinoma}

Overall, 29 patients underwent RNU for UTUC. Of these, $16(55 \%)$ and $13(45 \%)$ were treated in 2019 and 2020, respectively (Suppl. Table 2). No difference in terms of preoperative nor postoperative characteristics was found when comparing patients treated before and during COVID-19 pandemic. No difference was reported with regard to the time between the indication for surgery (either at diagnosis or after diagnostic ureteroscopy) and RNU (Fig. 1H).

\section{Renal cancer}

Overall, 142 patients were treated with either PN (92\%) or RN (8\%). Of these, 69 (49\%) and 73 (51\%) were treated in 2019 and 2020, respectively. No increase in the rate of RNs was seen in 2020. No differences in terms of pathological features or time between diagnosis and surgery were reported (Suppl. Table 3 and Fig. 1E, F).

\section{Testicular cancer}

Overall, 41 patients underwent orchifunicolectomy for testicular cancer. Of these, $24(58 \%)$ and $17(42 \%)$ were treated in 2019 and 2020, respectively. No differences in terms of pathological features were reported. Median time between diagnosis and surgery always remained below 2 weeks (Suppl. Table 4).

\section{Discussion}

With the prolongation of COVID-19 crisis, recommendations have been published to guide the urologists in the management of urological conditions, identifying four levels of priority. Depending on the resources and capacity, surgical treatment was recommended only for high-priority and emergency cases during COVID-19 pandemic, while intermediate-priority cases were considered only outside the COVID-19 surge [4]. Surgery was reported to be harmful in asymptomatic patients who subsequently tested COVID-19 positive [9], while older patients with comorbidity and cancer were found to be at higher risk of COVID-19 infection, severe manifestation of the disease, and fatal outcome [10].

As for elective uro-oncologic procedures, most of them were found to be safely postponed, or even changed to another treatment modality, given a limited availability of healthcare resources [7]. The main concern in delaying uro-oncological surgeries resides in the risk of cancer progression, and a potentially significant backlog of patients in need of cancer care, given the high incidence of these neoplasms [6]. As shown in a previous study conducted by our group, the cumulative delay in consultations and surgeries could have a ripple effect on future patients, further exacerbating potential adverse outcomes [3]. The risk of cancer progression obviously varies according to the type and grade of cancer, as highlighted by a recent collaborative review by Wallis et al. [1]. According to their results, treatment of most patients with intermediate- and high-risk PCa can be deferred 3-6 months without significant change in outcomes [11], while active surveillance should be the 

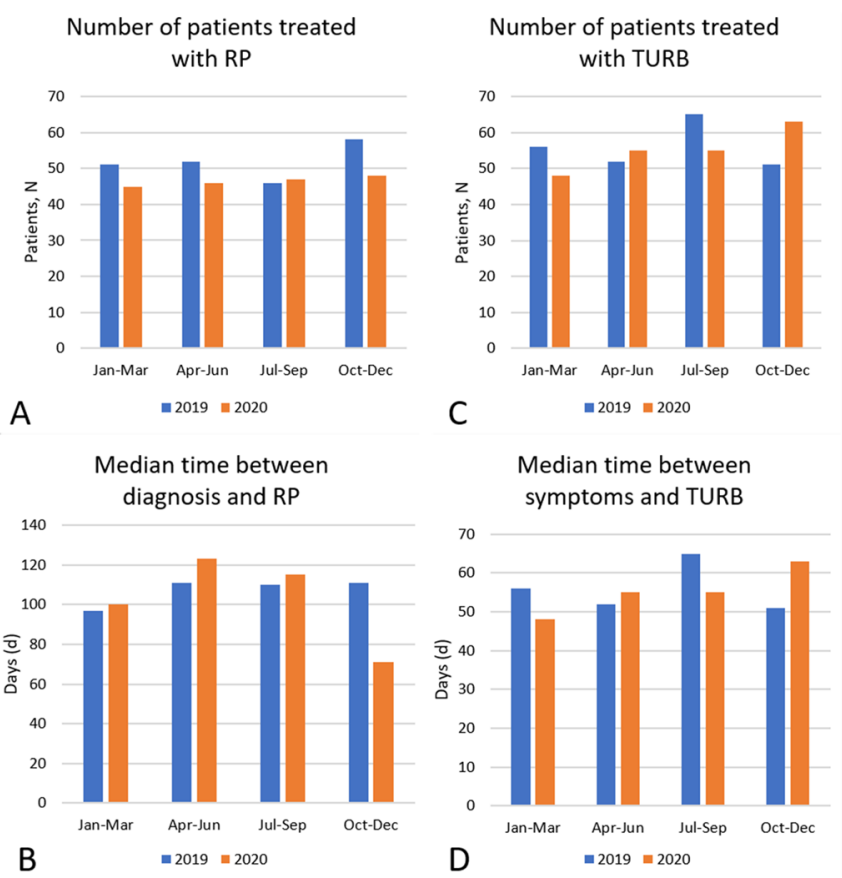

Fig. 1 Number of patients treated with RP (A), TURB (C), RN/PN $(\mathbf{E}), \mathrm{RC}(\mathbf{G})$, NUT $(\mathbf{H})$ for oncologic reasons. Median time between diagnosis and RP $(\mathbf{B})$, between symptoms and TURB (D), between diagnosis and RN/PN (F). Analyses per trimester. $R P$ radical pros-

preferred modality for low-risk PCa; the value of neoadjuvant androgen deprivation therapy is questionable and is not recommended. Patients with low-grade non-muscle-invasive $\mathrm{BCa}$ are unlikely to suffer from a 3-6 months delay, even if a re-evaluation is advised in case of new symptoms; on the contrary, risk of progression is seen for muscle-invasive $\mathrm{BCa}$ with RC delays beyond 12 weeks from diagnosis or completion of neoadjuvant chemotherapy. For patients with high-grade UTUC, delays of 12 weeks in RNU do not seem to be associated with adverse survival outcomes. As for renal tumors, surgery may be safely deferred for $\mathrm{T} 1 / \mathrm{T} 2$ renal masses, while locally advanced tumors should be treated expeditiously [1]. Surgical delay should be avoided when testicular cancer is suspected, also considering that orchifunicolectomy is typically performed in day-hospital and give a minimal burden on healthcare system [1].

During the COVID-19 pandemic, our regional health system was reorganized to create "COVID-19 hospitals" for the acute management of COVID-19 patients, while relieving this burden (or at least a part of it) for regional cancer referral centers like ours. It is our belief that the centralization of the uro-oncological activity in referral centers is essential to guarantee safe and high-quality treatments, and even more in time of crisis such as the COVID-19 pandemic.

Despite our efforts in maintaining our uro-oncological activity during COVID-19 pandemic, we would have expected a marked decrease in the number of our procedures
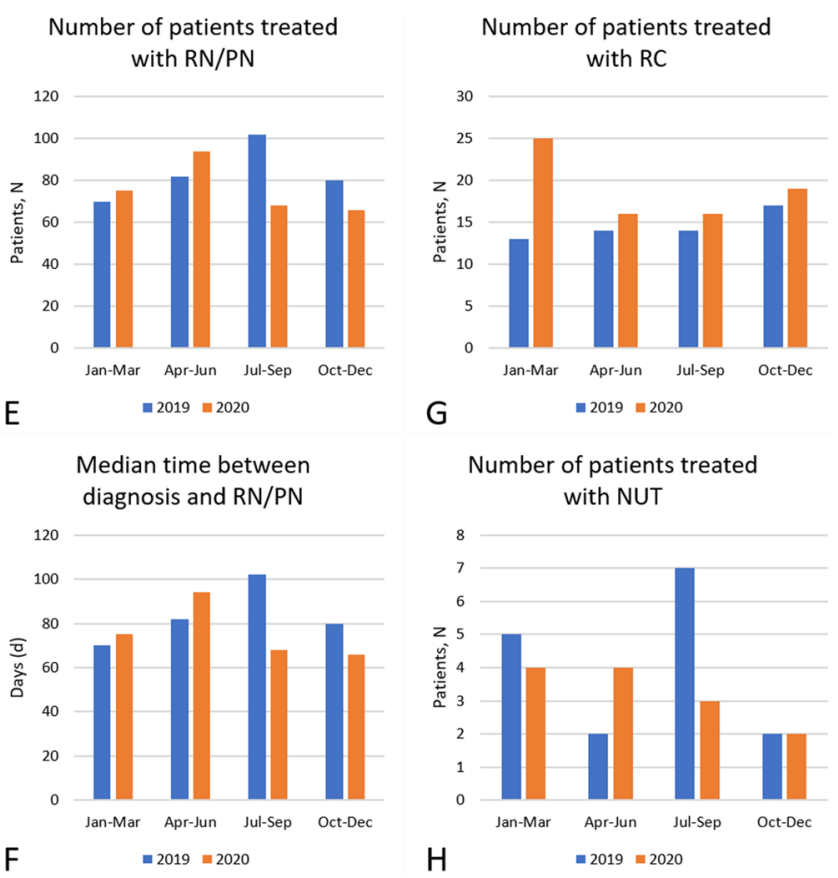

tatectomy, TURB trans-urethral resection of bladder, $R N$ radical nephrectomy, $P N$ partial nephrectomy, $R C$ radical cystectomy, $N U T$ radical nephroureterectomy

performed in 2020, as compared to 2019. On the contrary, no substantial decline was observed in our activity, likely as a consequence of our role as referral center, where urgent and oncologic procedures were allowed even during the pandemic. More importantly, no delay between diagnosis/ indication and surgery was found, as compared to the procedures of 2019. We were surprised by the low rate of patients whose surgeries were postponed due to COVID-19 positivity at preoperative workup. Unfortunately, we were not able to retrieve the data about a potential COVID-19 infection before preoperative workup, which might have caused a delay in the treatment. Following the recommendations of the European Association of Urology (EAU) [4, 5], priority was given to RC, which were all performed within 30 days from the indication or the completion of neoadjuvant chemotherapy (that was administered when indicated, notwithstanding the COVID-19). Locally advanced renal tumors, high-risk $\mathrm{PCa}$, and high-grade UTUC were also expeditiously treated. A mild decline in the number of TURBs was observed, probably because of the deferral of in-office surveillance cystoscopies of patients with known low-grade $\mathrm{BCa}$. While respecting the priority for the treatment of highrisk cancers, however, we continued to treat all urological neoplasms, as a tertiary referral center.

The most interesting results of the present study reside in the pathologic data resulting from our procedures: we had hypothesized a shift towards more advanced disease 
Table 2 Descriptive characteristics for the cohort of 767 patients who underwent trans-urethral resection of the bladder between Jan 2019 and Dec 2020

\begin{tabular}{|c|c|c|c|c|}
\hline \multirow[t]{2}{*}{ Variables } & \multicolumn{3}{|c|}{ Year of TURB } & \multirow[t]{2}{*}{$p$ value } \\
\hline & Total & 2019 & 2020 & \\
\hline Number of patients & 767 & $407(53)$ & $360(47)$ & \\
\hline Median age (IQR), years & $73(66-80)$ & $73(67-80)$ & $73(66-80)$ & 0.8 \\
\hline \multicolumn{5}{|l|}{ Gender, $n(\%)$} \\
\hline Female & 154(20) & $86(21)$ & $68(19)$ & \multirow[t]{2}{*}{0.4} \\
\hline Male & $613(80)$ & $321(79)$ & $292(81)$ & \\
\hline \multicolumn{5}{|l|}{ Primary vs recurrent tumor, $n(\%)$} \\
\hline Primary & $393(51)$ & $209(51)$ & 184(51) & \multirow[t]{2}{*}{0.9} \\
\hline Recurrent & $374(49)$ & 198(49) & $176(49)$ & \\
\hline \multicolumn{5}{|l|}{ Pathological tumor stage, $n(\%)$} \\
\hline pT0 & $84(11)$ & $46(11)$ & $38(11)$ & \multirow[t]{5}{*}{0.4} \\
\hline $\mathrm{pTa}$ & 291(38) & $148(37)$ & $143(40)$ & \\
\hline pTis & $8(1)$ & $2(0.5)$ & $6(2)$ & \\
\hline pT1 & 298(39) & $165(41)$ & 133(37) & \\
\hline pT2 & $86(11)$ & $46(11)$ & $40(11)$ & \\
\hline \multicolumn{5}{|l|}{ Pathological tumor grade, $n(\%)$} \\
\hline Low grade & $195(28)$ & $95(26)$ & $100(31)$ & \multirow[t]{2}{*}{0.2} \\
\hline High grade & 492(72) & $269(74)$ & 223(69) & \\
\hline Concomitant CIS, $n(\%)$ & $40(5)$ & $20(5)$ & $20(6)$ & 0.7 \\
\hline Lymphovascular invasion, $n(\%)$ & $40(6)$ & $18(5)$ & $22(7)$ & 0.09 \\
\hline Histological variants, $n(\%)$ & $72(9)$ & $29(7)$ & $43(12)$ & 0.02 \\
\hline \multicolumn{5}{|l|}{ Reason for performing TURB, $n(\%)$} \\
\hline Hematuria & $273(36)$ & $145(36)$ & $128(36)$ & \multirow[t]{4}{*}{0.9} \\
\hline Incidental diagnosis & $125(16)$ & $64(16)$ & $61(17)$ & \\
\hline Other symptoms & $60(8)$ & $32(8)$ & $28(8)$ & \\
\hline Recurrence at follow-up & $309(40)$ & $166(41)$ & $143(40)$ & \\
\hline $\begin{array}{l}\text { Median time from diagnosis to treat- } \\
\text { ment, days (IQR) }\end{array}$ & $56(34-82)$ & $55(35-82)$ & $56(34-83)$ & 0.8 \\
\hline
\end{tabular}

features, especially during the last months of 2020, as a consequence of late diagnoses. Nevertheless, no significant differences in terms of main pathologic features were observed in patients who underwent RARP, TURB, RNU and radical/ partial nephrectomy, probably because of the lack of surgical delay. A shift towards more aggressive disease was seen for prostate biopsy, where a higher proportion of ISUP grade 3 and 4 was diagnosed in 2020. This is hardly due to a deferral in urological consultations or PSA dosage, considering the long natural history of $\mathrm{PCa}$; more likely, it can be associated to the increase in fusion biopsies, which increase the diagnostic accuracy especially for cancers at high cellularity [12, 13]. The only cancer where more advanced disease features were seen at surgery, such as lymph node involvement and non-organ confined disease, was $\mathrm{BCa}$ with indication for $\mathrm{RC}$. This might reflect the aggressiveness of high-risk and muscle-invasive urothelial cancer, supporting once more the need for a urgent treatment in all circumstances. The management of testicular cancer deserves a separate chapter, as it must be promptly treated at all times, and minimally impacts on the healthcare system as orchifunicolectomy is a quick procedure that requires a 1-day hospitalization. In line with these considerations, in our center, we did not observe any differences in terms of pathological features or surgical delay between 2019 and 2020 .

This study is not devoid of limitations, mainly due to its monocentric design that might limit the generalizability of pathologic and referral trends. Furthermore, the short time span of study might hamper the evaluation of the effects of delayed screening due to COVID-19.

\section{Conclusion}

The volume of our uro-oncologic activity remained substantially stable between 2019 and 2020. Noteworthy is the absence of substantial delay in the treatment of uro-oncological diseases at our institution during the first year of COVID-19 pandemic. Importantly, no significant worsening of cancer disease features was found in 2020 except for muscle-invasive $\mathrm{BCa}$, which requires a prompt treatment. However, the risk of submerged disease and late diagnoses 
Table 3 Descriptive characteristics for the cohort of 134 patients who underwent radical cystectomy between January 2019 and December 2020

\begin{tabular}{|c|c|c|c|c|}
\hline \multirow[t]{2}{*}{ Variables } & \multicolumn{3}{|c|}{ year of radical cystectomy } & \multirow[t]{2}{*}{$p$ value } \\
\hline & Total & 2019 & 2020 & \\
\hline Number of patients & 134 & $58(43)$ & $76(57)$ & \\
\hline Median age (IQR), years & $73(65-78)$ & $73(64-77)$ & $73(66-79)$ & 0.6 \\
\hline \multicolumn{5}{|l|}{ Gender, $n(\%)$} \\
\hline Female & $33(25)$ & $17(30)$ & $16(21)$ & \multirow[t]{2}{*}{0.3} \\
\hline Male & 101(75) & $41(70)$ & $60(79)$ & \\
\hline BMI, median (IQR) & $26(23-28)$ & $25(23-27)$ & $26(23-28)$ & 0.7 \\
\hline \multicolumn{5}{|l|}{ Primary vs recurrent tumor at RC, $n(\%)$} \\
\hline Primary & $71(53)$ & $30(52)$ & $41(54)$ & \multirow[t]{2}{*}{0.8} \\
\hline Recurrent & $63(47)$ & $28(48)$ & $35(46)$ & \\
\hline \multicolumn{5}{|l|}{ Reason for performing RC, $n(\%)$} \\
\hline $\begin{array}{l}\text { Very high-risk NMIBC/BCG unrespon- } \\
\text { sive NMIBC }\end{array}$ & $43(32)$ & $19(33)$ & $24(32)$ & \multirow[t]{2}{*}{0.9} \\
\hline Muscle-invasive bladder cancer & $91(68)$ & $39(67)$ & $52(68)$ & \\
\hline Neoadjuvant chemotherapy, $n(\%)$ & $29(22)$ & $14(24)$ & $15(20)$ & 0.5 \\
\hline Preoperative hydronephrosis, $n(\%)$ & $41(31)$ & $16(28)$ & $25(33)$ & 0.5 \\
\hline \multicolumn{5}{|l|}{ Pathological tumor stage, $n(\%)$} \\
\hline pT0 & $14(10)$ & $6(10)$ & $8(11)$ & \multirow[t]{7}{*}{0.2} \\
\hline $\mathrm{pTa}$ & $5(4)$ & $1(2)$ & $4(5)$ & \\
\hline pTis & $6(4)$ & $3(5)$ & $3(4)$ & \\
\hline pT1 & $15(11)$ & $11(19)$ & $4(5)$ & \\
\hline pT2 & $15(11)$ & $7(12)$ & $8(11)$ & \\
\hline pT3 & $39(29)$ & $17(29)$ & $22(29)$ & \\
\hline pT4 & $40(30)$ & $13(22)$ & $27(38)$ & \\
\hline Non-organ confined disease, $n(\%)$ & $88(66)$ & $32(55)$ & $56(74)$ & 0.025 \\
\hline \multicolumn{5}{|l|}{ Pathological tumor grade, $n(\%)$} \\
\hline Low grade & $4(3)$ & $1(2)$ & $3(4)$ & \multirow[t]{2}{*}{0.5} \\
\hline High grade & $116(94)$ & $51(98)$ & $65(96)$ & \\
\hline Concomitant CIS, $n(\%)$ & $58(43)$ & $20(34)$ & $38(50)$ & 0.07 \\
\hline Lymphovascular invasion, $n(\%)$ & $72(54)$ & $26(45)$ & $46(61)$ & 0.07 \\
\hline Histological variants, $n(\%)$ & $45(34)$ & $20(34)$ & $25(33)$ & 0.8 \\
\hline \multicolumn{5}{|l|}{ Nodal tumor stage, $n(\%)$} \\
\hline No & $98(73)$ & $49(84)$ & $49(64)$ & \multirow[t]{2}{*}{0.01} \\
\hline $\mathrm{N}+$ & $36(27)$ & $9(16)$ & $27(36)$ & \\
\hline \multicolumn{5}{|l|}{ Urinary diversion, $n(\%)$} \\
\hline Ureterocutaneostomy & $9(7)$ & $4(7)$ & $5(7)$ & \multirow[t]{3}{*}{0.6} \\
\hline Ileum conduit & $82(63)$ & $39(67)$ & $43(59)$ & \\
\hline Orthotopic neobladder/ileal pouch & $40(31)$ & $15(26)$ & $25(34)$ & \\
\hline
\end{tabular}

cannot be ruled out, and might become apparent in a longer time span.

Supplementary Information The online version contains supplementary material available at https://doi.org/10.1007/s00345-021-03842-y.

Acknowledgements We thank Dr. Omar Kakaa, Dr. Simone Livoti, Dr. Federico Lavagno, Dr. Matteo Rosazza, and Dr. Ilaria Ferro for their valuable contribution in data acquisition.

Author contributions MO: project development, data analysis, and manuscript writing. FS: project development, data analysis, and manuscript writing. FR: data collection and data analysis. GC: data collection and manuscript editing. SM: data collection. AG: data collection. GP: data collection. AM: data collection. MDB: data collection. FV: data collection. EV: data collection. FP: data collection. GM: data collection. PG: project development and manuscript editing.

Funding The authors received no financial support for the research, authorship, and/or publication of this article.

Data availability Original data can be requested directly to the corresponding author.

Code availability Not applicable. 


\section{Declarations}

Conflict of interest The authors declare that there is no conflict of interest.

Ethical approval The procedures performed in this work were in accordance with the ethical standards of the institutional and/or national research committee and with the 1964 Helsinki Declaration and its later amendments or comparable ethical standards. The need of Institutional Ethics Committee approval was waived due to retrospective nature of the study, using only aggregate data.

Consent to participate All patients signed an informed consent for retrospective use of anonymized data for research purposes.

Consent for publications All patients signed an informed consent for retrospective use of anonymized data for research purposes.

\section{References}

1. Wallis CJD, Novara G, Marandino L, Bex A, Kamat AM, Karnes $\mathrm{RJ}$ et al (2020) Risks from deferring treatment for genitourinary cancers: a collaborative review to aid triage and management during the COVID-19 pandemic. Eur Urol 78(1):29-42

2. Oderda M, Roupret M, Marra G, Merseburger AS, Oderda G, Falcone $\mathrm{M}$ et al (2020) The impact of COVID-19 outbreak on uro-oncological practice across Europe: which burden of activity are we facing ahead? Eur Urol 78(1):124-126

3. Oderda M, Calleris G, Falcone M, Fasolis G, Muto G, Oderda G et al (2021) How uro-oncology has been affected by COVID-19 emergency? Data from Piedmont/Valle d'Aosta oncological network Italy. Urologia 88(1):3-8

4. Ribal MJ, Cornford P, Briganti A, Knoll T, Gravas S, Babjuk M et al (2020) European association of urology guidelines office rapid reaction group: an organisation-wide collaborative effort to adapt the European association of urology guidelines recommendations to the coronavirus disease 2019 era. Eur Urol 78(1):21-28

5. Heldwein FL, Loeb S, Wroclawski ML, Sridhar AN, Carneiro A, Lima FS et al (2020) A systematic review on guidelines and recommendations for urology standard of care during the COVID19 pandemic. Eur Urol Focus 6(5):1070-85

6. Mian BM, Siddiqui S, Ahmad AE (2021) Management of urologic cancers during the pandemic and potential impact of treatment deferrals on outcomes. Urol Oncol 39(5):258-267

7. Campi R, Amparore D, Capitanio U, Checcucci E, Salonia A, Fiori $\mathrm{C}$ et al (2020) Assessing the burden of nondeferrable major uro-oncologic surgery to guide prioritisation strategies during the COVID-19 pandemic: insights from three Italian high-volume referral centres. Eur Urol 78(1):11-15

8. Quarto G, Grimaldi G, Castaldo L, Izzo A, Muscariello R, De Sicato $S$ et al (2020) Avoiding disruption of timely surgical management of genitourinary cancers during the early phase of the COVID-19 pandemic. BJU Int 126(4):425-427

9. Lei S, Jiang F, Su W, Chen C, Chen J, Mei W et al (2020) Clinical characteristics and outcomes of patients undergoing surgeries during the incubation period of COVID-19 infection. EClinicalMedicine 21:100331

10. Liang W, Guan W, Chen R, Wang W, Li J, Xu K et al (2020) Cancer patients in SARS-CoV-2 infection: a nationwide analysis in China. Lancet Oncol 21(3):335-337

11 Diamand R, Ploussard G, Roumiguié M, Oderda M, Benamran D, Fiard G et al (2020) Timing and delay of radical prostatectomy do not lead to adverse oncologic outcomes: results from a large European cohort at the times of COVID-19 pandemic. World J Urol. https://doi.org/10.1007/s00345-020-03402-w

12. Kasivisvanathan V, Rannikko AS, Borghi M, Panebianco V, Mynderse LA, Vaarala MH et al (2018) MRI-targeted or standard biopsy for prostate-cancer diagnosis. N Engl J Med 378(19):1767-77

13. Savin Z, Dekalo S, Marom R, Barnes S, Gitstein G, Mabjeesh NJ et al (2021) The effect of delaying transperineal fusion biopsy of the prostate for patients with suspicious MRI findings-implications for the COVID-19 era. Urol Oncol gennaio 39(1):73.e1-73. e8

Publisher's Note Springer Nature remains neutral with regard to jurisdictional claims in published maps and institutional affiliations. 